\title{
A Política das Práticas Integrativas e Complementares do SUS: o relato de experiência sobre a implementação em uma unidade de ensino e serviço de saúde
}

\author{
sus integrative and complementary practices politics: report of \\ implementation in an educational and health care service
}

\author{
Aline Fernanda Fischborn, ${ }^{1}$ Juliane Machado, ${ }^{1}$ Naiele da Costa Fagundes, ${ }^{1}$ Natália Medeiros Pereira ${ }^{1}$ \\ 'Universidade de Santa Cruz do Sul (Unisc). Santa Cruz do Sul, RS, Brasil.
}

Recebido em: 21/09/2016 / Aceito em: 26/09/2016 / Publicado em: 18/10/2016

costanaiele@gmail.com

\section{RESUMO}

Percebendo que a Política das Práticas Integrativas e Complementares do SUS ainda é desconhecida por muitos profissionais de saúde, realizamos o relato de experiência da aplicação das práticas integrativas e complementares em uma Unidade de Ensino e Serviço de Saúde. Objetivo: avaliar os desafios da aplicação dessas práticas na unidade e analisar a percepção dos usuários e da equipe de saúde, frente a essas práticas. Método: se trata de um estudo descritivo do tipo relato de experiência, de caráter qualitativo, realizado em uma Unidade de Ensino e Serviço de Saúde em uma Universidade do interior do estado do Rio Grande do Sul, no período de abril a junho do ano de 2016, totalizando 28 pessoas envolvidas na implementação das práticas. Resultados: na prática da aromaterapia, utilizando óleos florais, três usuários negaram-se a participar, e os demais usuários, parabenizaram o trabalho, relatando sentir uma melhora no bem-estar físico. A técnica musicoterapia foi aplicada a 3 usuários, que relataram que o ambiente proporcionou calma e alívio da tensão nervosa que sentiam. Na aplicação da musicoterapia e aromaterapia aos membros da equipe, estes relataram a respiração leve, eliminação de tensão nervosa e sensação de bem-estar físico e mental. Já na capacitação sobre chás, houveram diversas perguntas sobre os benefícios e efeitos adversos do seu uso. Dos 28 indivíduos envolvidos nas práticas, 25 demonstraram interesse em conhecer as terapias integrativas e complementares. Considerações finais: constatamos resultados positivos aos indivíduos que se propuseram a participar das técnicas, como bem-estar físico, mental e alívio de tensão.
Por ser uma área nova de atuação para os profissionais de saúde, ainda há uma certa resistência, porém, trata-se de uma importante ferramenta na busca pela prevenção, promoção e tratamento dos usuários de saúde de forma integral, tal como prevê o SUS.

Palavras-chave: Integralidade em Saúde; Política de Saúde; Terapias Complementares.

\section{ABSTRACT}

Objective: realizing that the policy of Integrative and Complementary Practices of the Brazilian Unified Health System is still unknown by many disseminators health workers, we decided to run the application experience report of complementary and integrative practices in a Teaching Unit and Health Services, a university in the state of Rio Grande do Sul. The study objectives were to evaluate the challenges of application of these practices in the unit and analyze the perception of users and health team, against these practices. Method: a descriptive study of experience report characteristics, qualitative, conducted in a unit of Education and Health Services at a university in the interior of Rio Grande do Sul state, in the period from April to June of 2016, with 8 members of staff, 5 students and 15 users of the health service unit, totaling 28 people involved in the implementation of practices. Results: in the practice of aromatherapy, using floral oils, three users refused to participate, and other users, congratulated the work, reporting feel an improvement in the physical well-being. Music therapy technique (sound system) was applied to 3 users, who reported that the environment provi- 
ded calm and relieve nervous tension they felt. In the application of music therapy and aromatherapy to team members, they reported a slight breathing, elimination of tension and sense of physical and mental well-being. Of the 28 individuals involved in the practices, 25 showed interest in knowing the complementary and integrative therapies. Closing remarks: we found positive results for individuals who proposed to participate in techniques such as physical well-being, mental and stress relief. We understand that to be a new area of work for health professionals and health services, although there is some resistance, but it is an important tool in the search for prevention, promotion and treatment of health users in full, as required by the Unified Health System.

Keywords: Completeness Health; Health Policy; Complementary Therapies.

\section{INTRODUÇ̃̃O}

As Práticas Complementares, ainda que tenham surgido na antiguidade, foram redescobertas na atualidade pelo Ocidente, acompanhando a mudança do paradigma do modelo de atenção à saúde. Tais práticas defendem o cuidado integral ao paciente, atentando para a tríade corpo-mente-alma. Nos últimos anos, houve um interesse crescente pelas práticas complementares mundialmente em vários seguimentos da sociedade, incluindo os profissionais de saúde, que voltaram sua atenção para essas novas práticas.

Nesse sentido, o Programa de Medicina Tradicional foi desenvolvido em meados da década de 70 pela Organização das Nações Unidas, e desse período em diante, nasce um novo olhar na saúde que se volta aos processos de implementação terapêutica, a qual se denomina a Proposta da Política Nacional de Medicina Natural e Práticas Complementares. Na Conferência de Alma-Ata em 1978, a Organização Mundial de Saúde recomendou formalmente a utilização dos meios práticos da medicina tradicional/convencional pelos Sistemas de Saúde.

No ano de 2003, iniciou-se em nosso país a construção da Política Nacional de Práticas Integrativas e Complementares (PNPIC) no Sistema Único de Saúde, aprovada em fevereiro de 2006 pela Organização Mundial de Saúde. As terapias Integrativas e Complementares vieram como forma de reforçar o pressuposto da Integralidade na atenção à saúde, vendo o usuário de forma holística, diferenciado das terapias convencionais, que focam, em sua grande parte, em um determinado órgão a ser recuperado e que consideram a doença apenas sob o aspecto físico.

Assim, a Política Nacional de Práticas Integrativas e Complementares tem como objetivo desenvolver uma sistemática de atenção básica a qual vise estimular e desenvolver a promoção, prevenção e recuperação da saúde, podendo utilizar como recurso os métodos naturais, embasados no acolhimento e na criação de vínculos entre usuário, família e profissional de saúde. ${ }^{1}$ São portanto, práticas que auxiliam de forma complementar os tratamentos médicos, proporcionando ao paciente uma nova forma terapêutica, menos invasiva. ${ }^{2}$ São conhecidas também como terapias naturais, terapias complementares, terapias alternativas ou terapias integrativas. $^{1}$
É possível que, a cada ano, as indicações para a adesão ao tratamento, utilizando as práticas Integrativas e Complementares aumentem de forma considerável, uma vez que no Brasil, 232 municípios e 19 capitais já oferecem nos seus serviços de saúde alguma Prática Integrativa e Complementar aos seus pacientes. Com o apoio e incentivo do Governo Federal, as informações desta política de saúde estão em evidência nas Instituições de saúde do país.

A aceitação das práticas alternativas está em crescente ascensão no sistema de saúde, com técnicas como o Reiki, fitoterápicos, homeopatia, cromoterapia, acupuntura, meditação, massoterapia, musicoterapia, aromaterapia, entre outras. Porém, alguns profissionais de saúde estão despreparados para desenvolver essas novas técnicas devido à falta de conhecimento desta temática e o despreparo ao longo da formação. ${ }^{3}$

Dessa forma, o incentivo através do Ministério da Saúde e a tendência mundial de aumentar o debate acerca das práticas integrativas e complementares, impõem alguns desafios aos serviços de saúde, aos trabalhadores e aos graduandos da área da saúde. Embora haja uma normatização das ações de saúde, pautada inclusive pelos pressupostos do Sistema Único de Saúde, sobre o cuidado integral ao ser humano, sabe-se que na prática a operacionalização desse pressuposto enfrenta dificuldades. Ainda que alguns profissionais da saúde estejam procurando aperfeiçoamento para aplicar essas práticas no seu ambiente de trabalho, é notório que há uma lacuna na formação desses trabalhadores no que tange essa Política. ${ }^{4}$

Durante o período da graduação, é possível adquirir os conhecimentos mínimos necessários sobre as práticas integrativas e complementares, seus métodos, forma de aplicação, resultados e objetivos, para que assim, os futuros profissionais possam desenvolvê-las em seu ambiente de trabalho. Quando há falhas na formação dos graduandos, resultando na ausência de discussões sobre a Política Nacional de Práticas Integrativas e Complementares, há um comprometimento da própria aplicação da política nos serviços de saúde. ${ }^{3}$

Dessa forma, por um lado, percebendo a importância das práticas integrativas e complementares, e por outro, observando a lacuna de discussão dessas práticas no meio acadêmico, justifica-se a realização deste relato de experiência, que versa sobre a aplicação de duas práticas integrativas, a musicoterapia e a aromaterapia em Unidade de Ensino e Serviço de Saúde, em uma Universidade do interior do estado do Rio Grande do Sul.

Portanto, os objetivos desse trabalho foram implementar duas práticas integrativas em uma unidade de ensino e serviço, avaliar os desafios da aplicação dessas práticas e analisar a percepção dos usuários e equipe frente a essas práticas.

\section{MÉTODO}

Para realizar esse relato de experiência, utilizamos o apoio teórico de estudos descritivos, que exploram e identificam uma situação, serviço ou lugar conhecido, além da utilização do método qualitativo, que se caracteriza como um meio de compreensão, contribuição e 
análise dos indivíduos estudados. ${ }^{5}$

O estudo foi realizado em uma Unidade de Ensino e Serviço de Saúde em uma Universidade do interior do estado do Rio Grande do Sul, no período de abril a junho de 2016. O local do estudo atende a comunidade acadêmica e comunidade em geral, contando com profissionais de áreas multidisciplinares e acadêmicos de enfermagem, psicologia, medicina e nutrição, desenvolvendo diversas ações com grupos de idosos, gestantes, adolescentes entre outros, e atendimentos individuas como pré-câncer, vacinas, curativos e consultas nas diversas especialidades. Os sujeitos desse estudo foram 8 funcionários, sendo 4 técnicos de enfermagem, 2 auxiliares administrativos, 2 enfermeiros; 5 acadêmicos, sendo 4 de enfermagem e 1 de psicologia, e 15 usuários do serviço de saúde da unidade, totalizando 28 pessoas envolvidas nas atividades.

A implementação das práticas se deu em duas frentes: dois momentos de capacitação, um com a equipe de funcionários, acadêmicos e usuários, e outro apenas com os funcionários do serviço; a aplicação das práticas com os usuários, no momento de seu atendimento na unidade, e com os funcionários do serviço, antes do início da sua jornada de trabalho.

\section{RESULTADOS}

O processo da capacitação sobre o uso e benefícios dos chás, aromaterapia e musicoterapia, foi aberto a comunidade tendo a participação de funcionários, acadêmicos e usuários em geral, totalizando 15 participantes. Já a segunda etapa de capacitação sobre a implantação da aromaterapia e musicoterapia, teve como público alvo os funcionários do serviço, totalizando 8 participantes. Neste período foram explanados aspectos como finalidades, benefícios, meios e métodos a serem utilizados para que a proposta de aplicação dessas terapias transcorresse de forma a beneficiar os usuários do serviço.

Nos momentos de capacitação sobre a Política Nacional das Práticas Integrativas e Complementares do SUS, os profissionais relataram já ter ouvido falar sobre o assunto, porém desconheciam sua proposta, bem como a sua finalidade. Os estudantes mostraram-se interessados sobre o assunto, e reivindicaram sobre a pouca abordagem deste tema na grade curricular. Os usuários por sua vez, se identificaram com o uso dos chás, além de relatarem sobre a importância de se saber a forma correta de utilização dos fitoterápicos.

No momento de relaxamento oferecido à equipe, que aconteceu uma vez por semana, foi utilizado o som ambiente e óleos florais colocados de forma tópica nas mãos dos funcionários, antes do início de trabalho. Estas técnicas também foram utilizadas em momentos de estresse, fadiga física e mental dos funcionários da equipe. Neste período, os participantes disseram sentir-se com respiração leve, eliminação de tensões nervosas e elevada sensação de bem-estar físico, mental e emocional.

A aplicação aos usuários da unidade aconteceu na sala de acolhimento, onde foram aplicadas a aromaterapia e a musicoterapia, sendo utilizados óleos florais de lavanda e menta nas mãos dos usuários que aceitaram participar das práticas, além do oferecimento de música ambiente, neste mesmo espaço. No momento desta prática, dois usuários negaram-se a participar, alegando que estavam com urgência de ir embora. Os demais usuários parabenizaram o trabalho, relatando sentir uma melhora no bem-estar físico e mental, além de um sentimento de paz interior, melhora no processo respiratório e corpo leve.

Por sua vez, na sala de coleta de exame cito patológico, foi utilizada a musicoterapia através da oferta de som ambiente ao longo do atendimento de 3 pacientes. Estas, relataram que o ambiente proporcionou calma e alívio da tensão e do nervosismo que sentiam no momento do exame. A música escolhida para o processo terapêutico foi a de som ambiental, no formato sonoro e calmo. A proposta desta música pode ser justificada por emitir sons de paz e sem potência vibratória ao ambiente, sendo que esta atua de forma direta no campo energético de cada pessoa, proporcionando equilíbrio entre o meio externo e interno de cada indivíduo.

\section{DISCUSSÃO}

\section{As Práticas Integrativas e Complementares}

É possível perceber duas linhas de seguimento na saúde: a visão biológica e a hegemonia dos médicos sob os demais profissionais, onde predominam os avanços tecnológicos na medicina e fortalecimento das atenções secundária e terciária. ${ }^{6}$ Em demais sistemas de saúde, como por exemplo o da medicina tradicional, o paradigma cartesiano biomédico tem menor prevalência. E a medicina moderna acaba por instigar questionamentos, no que se refere a atenção da atual demanda. ${ }^{7}$

Em 1970 a Organização Mundial da Saúde implementou o Programa de Medicina Tradicional, com objetivo de formular políticas nesta área, e desde aí vem demonstrando um compromisso pelo incentivo aos seus estados-membros, para que elaborem e instituam estas práticas de forma racional e integrada na atenção à saúde. Ressalta também a importância da realização de estudos e pesquisas na área, objetivando maior conhecimento sobre as práticas, como segurança na sua aplicação, efetividade e eficácia. ${ }^{8}$ No entanto, algumas mudanças vêm ocorrendo e sugerem uma interferência em processos e crenças já consolidados, dentre estas a construção da Política Nacional de Práticas Integrativas e Complementares do Sistema Único de Saúde no ano de 2006, fortaleceu e respaldou as práticas que já vinham sendo realizadas em alguns municípios e estados brasileiros, estabelecendo uma relação entre a medicina tradicional às práticas não convencionais. ${ }^{6,7}$

Estas práticas e saberes foram denominadas pela Organização Mundial da Saúde como Medicinas Complementares e Alternativas/Medicinas Tradicionais, nas quais o ser humano tem a oportunidade de integrar-se à natureza, onde há uma visão ampliada dos processos de saúde e adoecimento. A nível mundial, cerca de $80 \%$ dos países do hemisfério Sul praticam alguma forma de Medicina Tradicional Complementar em seus cuidados básicos de saúde. ${ }^{7}$ Dessa forma, as práticas estão tendo crescimento mundial ressaltando um importante aspecto: a legislação das práticas integrativas e complemen- 
tares apresenta respeito e reconhecimento, mostrando a conquista dos diferentes espaços existentes em nossa sociedade, na busca pelas práticas seguras, holísticas e igualitárias. ${ }^{9}$

Dentre os preceitos trazidos pela política, destacam-se o cuidado humanizado, integral e continuado, a prevenção de agravos, promoção e recuperação da saúde dos indivíduos. ${ }^{8}$ Estes, também fazem parte da lei número 8.080 , de 19 de setembro de 1990, que evidencia os princípios citados acima, além de reforçar também a importância de se garantir às pessoas o bem-estar físico, mental e social. ${ }^{10}$ Dessa forma, podemos perceber que os princípios da Política Nacional de Práticas Integrativas e Complementares condizem e reafirmam os conceitos trazidos pelo SUS.

Outro aspecto importante a destacar é o baixo custo que as práticas complementares apresentam. Além de seus inúmeros benefícios, ainda promovem a redução de gastos o que se faz importante para o nosso sistema de saúde, na medida em que alia uma maior eficácia na terapêutica de algumas patologias com viabilidade econômica. ${ }^{7}$

Porém, mesmo com o apoio e respaldo da Política Nacional, a implementação das práticas passa por algumas limitações, como a dificuldade de adoção às práticas pela diferença em relação ao modelo biologicista. Percebe-se também, que no que diz respeito ao conhecimento em relação à política ainda há algumas falhas. É possível identificar em um estudo realizado em dois serviços da Prefeitura Municipal de São Paulo que nem todos os profissionais atuantes tinham conhecimento sobre a Política Nacional, somente sobre algumas práticas, como por exemplo a homeopatia e a acupuntura. ${ }^{7}$

Em nosso estudo, também foi identificada esta fragilidade de informação, em que os profissionais tinham um certo conhecimento sobre a temática, mas não conheciam suas reais finalidades. Identificou-se também que os profissionais da unidade se mostraram satisfeitos após a realização das práticas, relatando sentirem mudanças em seu estado físico, emocional e mental.

No mesmo estudo realizado na Prefeitura Municipal de São Paulo, os profissionais também faziam o uso de algumas práticas, e em sua maioria acreditavam que há efetividade terapêutica no uso destas práticas, além de considerarem o seu uso excelente e acreditarem que os usuários do serviço precisam saber que, para se ter saúde, não é necessário somente o uso de medicações, além de relatarem que, após passarem por algumas práticas integrativas e complementares, houve a diminuição do estresse, maior tranquilidade e bem-estar físico e emocional. $^{7}$

Assim, ainda mesmo com avanços na área, ainda perduram desafios. Dentre esses, podemos citar a falta de conhecimento sobre a Política Nacional de Práticas Integrativas e Complementares por parte dos usuários e profissionais. O que foi encontrado no referencial teórico está em concordância com os resultados obtidos em nossa pesquisa, sendo possível destacar, como exemplo, a falta de conhecimento por parte dos profissionais, citada em nossos resultados. Alguns profissionais atuantes na saúde pública desconhecem a política e alguns tem conhecimento sobre algumas práticas, porém não compreendem o real objetivo da política. ${ }^{7}$
Segundo estudo realizado com equipes de saúde da família de Florianópolis, a maioria dos profissionais desconhece as diretrizes nacionais da Política Nacional de Práticas Integrativas e Complementares, porém, grande parte destes mesmos profissionais mostram-se favoráveis ao que a política propõe. ${ }^{10}$ Além disso, poucos profissionais participantes desta pesquisa, afirmaram ter pouco ou nenhum interesse em capacitar-se sobre esse assunto. ${ }^{11}$

Tal fato pode ser explicado por haver uma escassez de discussão sobre esse tema na formação dos futuros trabalhadores da saúde. No Brasil, há ainda a poucas instituições que aderem aos conceitos e técnicas trazidas pela Política Nacional de Práticas Integrativas e Complementares, sendo que uma das melhores formas de divulgação dessas práticas e política é a adesão de instituições de ensino, ofertando cursos de profissionalização na área ou disciplinas curriculares que estejam em harmonia com o que é respaldado pelo Sistema Único de Saúde, para que se fortaleça a aproximação entre ensino e serviço e minimize as chances de adoção do formato intervencionista e biologicista pelos serviços de saúde. ${ }^{6}$

Por sua vez, o Ministério da Saúde dispõe de cursos gratuitos oferecidos a profissionais já formados e usuários da rede, como o Curso Introdutório em Práticas Integrativas e Complementares: Antroposofia aplicada à Saúde e o Curso Introdutório em Práticas Integrativas e Complementares: Práticas corporais e mentais da Medicina Tradicional Chinesa, além de disponibilizar também no site do Ministério da Saúde Cadernos de Atenção Básica sobre plantas medicinais e fitoterapia, lançado no ano de 2012.

\section{Aromaterapia, Musicoterapia e o Uso de Chás}

Não há como afirmar a data inicial, em que a utilização de ervas para fins terapêuticos nasceu. Estas foram sendo conhecidas aos poucos, com o passar dos séculos. As autoras afirmam que, a partir de registros literários, em 2000 a.C., os sacerdotes egípcios, considerados os primeiros aromaterapeutas da história, utilizavam no processo de mumificação, óleos como o de cedro e outros aromáticos. ${ }^{12}$

O tratamento com florais deriva de extratos líquidos, sendo preparados na forma de infusão solar das flores. Quando prontas, as flores são colocadas em recipiente com água pura, posteriormente diluídas, potencializadas e conservadas. ${ }^{1}$ Esta prática objetiva o tratamento holístico de patologias físicas e emocionais, a partir da utilização de óleos essenciais, após a realização de uma análise que considere aspectos físicos, mentais e emocionais, avaliação e acompanhamento no período do tratamento. Estes óleos causam bem-estar aos indivíduos, além de auxiliarem na sua recuperação, e podem ser indicados para tratamentos cosméticos como acne, seborreia, caspa, tratamento de alterações como insônia, estresse e no tratamento de patologias, como asma, gota e depressão. ${ }^{12}$

Os óleos essenciais podem ser utilizados através de três vias: a inalação, a aplicação na pele e a ingestão. $\mathrm{Na}$ inalação, eles atravessam as vias respiratórias superiores em direção às inferiores, onde são então absorvidos pelos vasos sanguíneos pulmonares, 
e finalmente, agem em todo o sistema respiratório. $\mathrm{Na}$ aplicação cutânea, os óleos são absorvidos pela pele e mucosas, indo em direção a corrente sanguínea e por último, encaminham-se para os tecidos e órgãos. Quando são ingeridos, os óleos essenciais passam pelo sistema gastrointestinal, onde posteriormente são absorvidos nos intestinos, atingindo a corrente sanguínea, e em seguida, são encaminhados aos tecidos e órgãos do nosso corpo. O uso através da ingestão só pode ser recomendado pelo profissional médico, pois o organismo pode não responder de forma adequada às substâncias utilizadas. ${ }^{12}$

O estresse é capaz de causar desequilíbrios aos seres humanos, sendo a aromaterapia uma boa alternativa para melhorá-lo, uma vez que consegue diminuir os efeitos que essa patologia causa, atuando com seus princípios sedativos, calmantes e relaxantes, devendo levar-se em consideração, a necessidade de um profissional habilitado para execução desta técnica, a fim de se obter os efeitos esperados. ${ }^{13}$

Por sua vez, a musicoterapia está ganhando espaço nos serviços de saúde, podendo ser utilizada em diversas áreas de atuação, e devendo ser exercida por um profissional com formação específica, o musicoterapeuta. Mesmo sendo uma prática descoberta antigamente, acaba ganhando espaço para pesquisa e aplicação após a comprovação de seus diversos efeitos benéficos, tanto nos atendimentos feitos pelos profissionais, quanto na vida diária. ${ }^{14} \mathrm{~A}$ música atua no sistema nervoso autônomo, fazendo com que o paciente desvie sua atenção da dor, raiva, culpa e ódio, auxiliando na tentativa da diminuição do uso de medicamentos analgésicos e proporcionando momentos de descontração e divertimento. ${ }^{15}$

A música desenvolve uma alteração no humor dos pacientes que a escutam, seja antes, durante ou após os atendimentos, além de melhorar aspectos emocionais, físicos, mentais, espirituais e cognitivos, uma vez que ocorre o relaxamento e diminuição dos sentimentos de raiva, estresse, dor e tristeza, libera sensações de conforto e bem-estar para uma melhora na qualidade de vida. ${ }^{15}$

As instituições brasileiras credenciadas nas Associações Nacionais e Latino-Americana de Cuidados Paliativos, onde as equipes de funcionários escolheram a musicoterapia como estratégia de intervenção, por acreditarem que esta envolve o encontro com o outro. ${ }^{15}$ As autoras observaram que durante este momento, os usuários sentiam-se desrespeitados pelo atraso dos médicos, resultando em mau humor, cansaço, estresse, preocupação, ansiedade e perca de tempo. Após incluírem esta técnica, foi possível identificar que os usuários do serviço se sentiram mais leves, valorizados, calmos, tolerantes, com humor, saúde e bem-estar físico melhorados após a utilização da técnica. ${ }^{15,16}$

O uso dos chás se faz desde o início do ciclo da vida. As plantas medicinais classificam-se como uma forma de recurso totalmente natural, que tem o poder de potencializar a restauração da saúde e do bem-estar dos indivíduos. As plantas medicinais, conhecidas popularmente como chás, vem ganhando espaço no mundo científico, uma vez que inúmeros estudos estão sendo desenvolvidos para que a finalidade de comercialização e seu uso tenham ação terapêutica eficaz. No mesmo sentido, a Organização Mundial da Saúde permite a utilização dos chás, pois compreende como um apoio prático à medicina tradicional, porém considera que o seu uso requer a identificação e classificação botânica adequada com o intuito de evitar a atividade entre o princípio ativo das plantas. ${ }^{17}$

Os chás são comumente utilizados por pessoas da terceira idade e, desta forma, é fundamental o conhecimento acerca da forma correta de consumo destas plantas, pois nesta fase da vida, o ciclo vital está em declínio e, consequentemente, as funções metabólicas. Por isso, se faz necessário um cuidado especial nessa fase da vida, ainda que não se descarte esse mesmo cuidado nas demais faixas etárias da vida, tendo em vista que cada chá possui uma indicação terapêutica. ${ }^{18}$

Assim, em nosso estudo, identificamos que os usuários ao serem expostos a aromaterapia e a musicoterapia ficaram calmos, sentindo alívio da tensão e do nervosismo, além da melhora no bem-estar físico, mental e emocional. Da mesma forma, na capacitação sobre o uso de chás, houveram dúvidas sobre a maneira correta de utilização das plantas e interesse sobre os efeitos adversos do uso incorreto das mesmas.

\section{CONSIDERAÇÕES FINAIS}

Após a realização de pesquisa em referencial teórico, na busca por melhor compreensão sobre a Política Nacional de Práticas Integrativas e sua temática, e colocá-la em prática, constatamos que houveram resultados positivos e consideráveis às pessoas que se propuseram a participar das práticas.

Entendemos que por ser uma nova área de atuação dos profissionais de saúde, ainda não há resistências, porém, trata-se de uma importante ferramenta na busca pela prevenção, promoção e tratamento dos usuários de saúde entendendo o ser humano de forma integral e holística.

Em determinados momentos, os usuários que foram ao serviço de saúde em busca de atendimento, sentiram-se cansados, estressados e tensos. Após submeterem-se às técnicas naturais, notou-se os benefícios que estas são capazes de promover, a partir do relato destes usuários, com resultados positivos como melhora de bem-estar físico e mental.

Infelizmente destacamos a falta da abordagem da Política, tanto na formação dos profissionais de saúde, como nas unidades de saúde da região onde a universidade pesquisada se insere. Mesmo tendo respaldo de órgãos competentes e estudos científicos que comprovem sua eficácia, muitos profissionais alegam que já terem ouvido em algum momento sobre o tema, mas acabam não se qualificando pela falta de conhecimento sobre objetivos, finalidades, ou seja, o conteúdo da política.

Com isso, é pertinente dizer que cabe a todos os profissionais da saúde uma qualificação sobre a Política Nacional de Práticas Integrativas e Complementares, para então poder consagrá-la e usufruir de seus inúmeros benefícios que vem a atender não só aos pacientes, mas também os profissionais atuantes.

Ao final, acreditamos na importância da reformulação nas grades curriculares dos cursos na área da saúde, considerando a pouca abordagem sobre a Política Nacional e as PIC na formação dos estudantes da área 
da saúde. Assim será possível transmitir o real significado destas, além de auxiliar aos futuros profissionais que atuarão em nosso sistema público a desenvolverem esta temática com mais efetividade e qualidade.

\section{REFERÊNCIAS}

1. Brasil. Ministério da Saúde. Política Nacional das Práticas Integrativas e Complementares do SUS. 2008.

2. Santos LF, Cunha, AZS. A Utilização de Práticas Complementares por Enfermeiros do Rio Grande do Sul. Rev. enfermagem UFSM. 2011.

3. Silva NCM, lunes DH, Resck ZMR, Soares MI, Junior DIS, Vieira NF. Estratégia de Ensino das Terapias e Complementares na Graduação em Enfermagem /Revisão Integrativa. Rev. Eletrônica de Enfermagem. 2013.

4. Bastos RA, Silva LB, Lima IC. Terapias Complementares Integrativas: conhecimento e utilização pelos docentes do curso de enfermagem de uma instituição. Rev Saúde Col UEFS 2015;5(1):40-5.

5. Leopardi M T. Metodologia da pesquisa na saúde. Editora Pallotti. Santa Maria. 2001.

6. Azevedo E, Pelicioni MCF. Práticas Integrativas e Complementares de desafios para a educação. Trab Educ Saúde. Rio de Janeiro 2012;9(3):361-78. doi: 10.1590/ S1981-77462011000300002.

7. Ischkanian PC, Pelicioni MCF. Desafios das Práticas Integrativas e Complementares no SUS visando a promoção de saúde. Revista Brasileira de Crescimento Desenvolvimento Humano 2012;22(2):233-38. doi: 10.7322/jhgd.44936.

8. Brasil. Ministério da Saúde. Secretaria de Atenção à Saúde. Departamento de Atenção Básica. Política nacional de práticas integrativas e complementares no SUS: atitude de ampliação de acesso. 2. ed. Brasília: Ministério da Saúde. 2015.

9. De Simoni C, Benevides I, Nelson FB. As práticas integrativas e complementares no SUS: realidade e desafios após dois anos de publicação da PNPIC. Revista Brasileira de Saúde da Família. Ano IX, ed. espec. Brasília: Ministério da Saúde. 2008.

10. Brasil. Lei.8.080, de 19 de setembro de 1990. Dispõe sobre as condições para promoção, proteção e recuperação da saúde. Diário Oficial da União. Brasília, DF, 20 set. 1990. P 1855.

11. Thiago SCS, Tesser CD. Percepção de médicos e enfermeiros da Estratégia de Saúde da Família sobre Terapias Complementares. Revista de Saúde Pública 2011;45(2):249-57.

12. Gnatta JR, Dos Santos SD. Aromaterapia e Enfermagem. In: Salles LF, Silva MJP, organizadores. Enfermagem e as Práticas Complementares em Saúde. São Caetano do Sul: Yendis; 2011. p. 61-76.

13. Paganini T, Silva YF. O uso da aromaterapia no combate ao estresse. Arquivos de Ciências Unipar. Umuarama 2014;18(1):43-9.

14. Arndt AD, Volpi RC, Volpi S. Aspectos da prática musicoterapêutica: contexto social e comunitário em perspectiva. Psicol Soc. 2016;28(2):387-95. Doi: 10.1590/1807-03102016v28n2p387

15. Caires JS, Andrade TA, Amaral JB, Calasans ATA, Rocha MDS. A utilização das terapias complementares nos cuidados paliativos: benefícios e finalidades. Cogitare Enferm 2014;19(3):514-20. doi: 10.5380/ce.v19i3.33861

16. Pimentel AF, Barbosa RM, Chagas A. A musicoterapia na sala de espera de uma unidade básica de saúde: assistência, autonomia e protagonismo. Inteface - Comunicação, Saúde e Educação 2011;15(38):174-754.

17. Salles LF, SILVA MJ. Enfermagem e as práticas complementares em saúde. Editora Yendis. 2011. p. 98-101.

18. França ISX, Solza JA, Baptista RS, Britto VRS. Medicina popular: benefícios e malefícios das plantas medicinais. Rev. Bras. Enf. Brasília. 2008.

Como citar: FISCHBORN, Aline Fernanda et al. A Política das Práticas Integrativas e Complementares do SUS: o relato da im plementação em uma unidade de ensino e serviço de saúde. Cinergis, Santa Cruz do Sul, v. 17, out. 2016. ISSN 2177-4005. Disponível em: <https://online.unisc.br/seer/index.php/cinergis/article/view/8149>. Acesso em: 11 out. 2016. doi:http:// dx.doi.org/10.17058/cinergis. v17i0.8149. 